\title{
Hybrid Overlay/Underlay Cognitive Radio Network with MC-CDMA
}

Fahimeh Jasbi, Member, IEEE , and Daniel K.C. So, Senior Member, IEEE

\begin{abstract}
Cognitive radio (CR) allows secondary users (SUs) to exploit the under-utilized radio spectrum of the primary networks. To fully utilize the primary spectrum and maximize the spectral efficiency, overlay and underlay transmissions, which exploit the white and grey spaces respectively, should be used together. However for underlay, the SUs need to transmit at low power to avoid causing harmful interference to the PUs, whereas the PUs will cause high interference to the SUs. Thus interference mitigation is a major issue in underlay spectrum utilization. In this paper, a hybrid transmission system that exploits both overlay and underlay is proposed using MC-CDMA due to its interference rejection and diversity exploitation capabilities. Unlike existing approaches, which separate the use of overlay and underlay spectrum, the proposed schemes utilize the entire spectrum for underlay transmission to minimize the PU interference while using the spectrum holes for overlay transmission to maximize data rate. Two techniques that operates at full-load and overload scenarios are proposed. The overload system is then extended to the multi-user underlay case to further improve the spectrum utilization. Index Terms-Cognitive Radio (CR), Orthogonal Frequency Division Multiplexing (OFDM), Multi-Carrier Code Division Multiple Access (MC-CDMA), overlay and underlay spectrum access
\end{abstract}

\section{INTRODUCTION}

Cognitive Radio (CR) is a promising solution to the spectrum under-utilization problem by opportunistically accessing the spectrum of the primary network. There are two main spectrum access mechanisms in CR networks: overlay and underlay. Overlay transmission utilizes the spectrum holes by exclusively using the unoccupied spectrum of the Primary User (PU) and vacates on PU re-occupancy. On the other hand, underlay transmission can utilize the spectrum at any time by transmitting in the presence of PUs at low power to avoid causing harmful interference. The overlay and underlay CR approaches have been investigated widely in the literature [1]-[4]

In particular, different multiplexing or multiple access schemes have been proposed for the physical layer transmission of overlay CR systems. Orthogonal Frequency Division Multiplexing (OFDM) is a strong candidate for overlay due to its flexibility to fill in the spectrum holes non-contiguously, known as NC-OFDM [5]. However, a major drawback is the large side lobes that results in high out-of-band emission which can leak

Copyright (c) 2015 IEEE. Personal use of this material is permitted. However, permission to use this material for any other purposes must be obtained from the IEEE by sending a request to pubspermissions@ieee.org. into an active PU band and hence significantly degrade PU's performance [6]. Considering this interference, coexisting primary and secondary users in adjacent bands of an OFDM-based overlay system is investigated [7]. The framework is then extended to the case where different interference constraints are set by different PUs in [8]. The non-contiguous transmission approach is applicable to other multicarrier techniques, such as Multi-Carrier Code Division Multiple Access (MC-CDMA). Authors in [9] proposed an NC-MC-CDMA scheme that adaptively changes its transmission parameters according to the available spectrum holes instead of the sub-band deactivation method.

There has been a shift from the conventional transmitter-centric model by FCC Spectrum Policy Task Force [10] in 2002. The new model introduces interference threshold at the receiver side where interference takes place rather than interference being controlled at a certain distance from the transmitter [11]. This is to ensure that the CR system will not harm the licensee's performance. Therefore, underlay transmission is more challenging as utilizing the same spectra as PU may suffer from high interference and hence considerably degrade its performance. Thus, a major issue in underlay spectrum utilization is interference mitigation.

In recent years underlay transmission has been widely investigated in the literature. Yet, spread-spectrum-based techniques are preferable for underlay CR systems (see e.g. [12]). There are two fundamental advantages of spread spectrum systems to be utilized in underlay CR. The first stems from low power density due to spreading, and the second is the capability to mitigate high interference levels [10]. While existing literatures agree on utilizing spread-spectrum-based schemes for underlay due to their interference suppression capabilities [10], [12], [13], there is a missing link on how to achieve such interference suppression when all the bandwidth is either occupied by PU or the overlay SU. This is known as the hybrid CR system where both overlay and underlay are jointly exploited. The main purpose of this paper is to address this issue.

Previous works show that the hybrid systems outperform overlay or underlay only systems in terms of two important performance measures: total achievable transmission rate [14] and Bit Error Rate (BER) performance [15]. An OFDMA-based joint overlay and underlay spectrum access mechanism is proposed in 
[14]. A hybrid overlay/underlay transmission scheme was proposed for CR systems in AWGN channels in [16]. Overlay carries the modulated data utilizing NC-OFDM, while underlay carries parity bits using NC-MC-CDMA technique. The performance is also examined for fading channels in [15], assuming the received interference from the PU at SU to be passing through AWGN channel.

In this paper, we propose a hybrid CR system that fully utilizes all white and grey spaces in the spectrum using MC-CDMA. The PU signals are treated as narrowband interferences and are suppressed by the nature of MC-CDMA. In order to maximize spectrum efficiency, the white spaces should be occupied by overlay SUs, which will also cause interference to the underlay users. The overlay and underlay users are separated using different scrambling codes. We present a code allocation algorithm that ensures the underlay SUs can suppress PUs interference while having very low or zero cross correlations with overlay SUs. In particular, two hybrid approaches are proposed, namely the Full-Load and the Overload. Both systems are hybrid MC-CDMA systems which are flexible to any available number of spectrum holes. Two detection schemes are also proposed for the hybrid CR systems to mitigate the interferences. It is worth mentioning that although some of the techniques used in this work are available in the existing CDMA literature, applying the techniques in this way in CRN is new.

The following notations are used in the paper. Upper and lower-case boldface letters denote matrices and vectors respectively. $\otimes$ shows the Kronecker product. Moreover, (.) $)^{T},(.)^{*},(.)^{-1}$ and $(.)^{H}$ stand for matrix transpose, complex conjugate, matrix inversion and Hermitian transpose respectively. $\mathbf{X}=\operatorname{diag}\left[x_{1}, \ldots, x_{M}\right]$ is an $M$ by $M$ block diagonal matrix with the diagonal elements given by vector $\mathbf{x}=\left[x_{1}, \ldots, x_{M}\right]$. The trace of the diagonal matrix $\mathbf{X}$ is denoted by $\operatorname{tr}(\mathbf{X})$. Also $\mathbb{C}^{a \times b}$ defines the space of $a \times b$ with complex entries. Variable definitions also included in Table I.

The rest of this paper is as follows: Section II describes the systems model. The proposed Full-Load hybrid model is explained in Section III, followed by the modified chip-level and symbol-level MMSE equalizers. Section IV presents the proposed overload hybrid model and is then extended to a multi-user hybrid system. Simulation results are presented in Section V, and finally conclusions are drawn in Section VI.

\section{System MOdeL}

The spectral occupancy of the hybrid MC-CDMA system is shown in Fig. 1 where the primary OFDMA-based system and the Cognitive Radio Network (CRN) coexist in the same band, with bandwidth $B$ which is divided into $M$ equi-width subcarriers. It is assumed that spectrum sensing is performed using some accurate spectral sensing techniques (e.g., [17], [18]) perfectly and the available
TABLE I: Variable Definitions

\begin{tabular}{|l|l|}
\hline Variable & Definition \\
\hline $\mathbf{a}[i]$ & $i$-th subcarrier availability \\
$B$ & Total available bandwidth \\
$\overline{\mathbf{b}}[\bar{k}]$ & $k$-th overlay user's data vector \\
$\mathbf{b}[k]$ & $k$-th underlay user's data vector \\
$\overline{\mathbf{c}}[\bar{k}]$ & $k$-th overlay user's specific signature \\
$\mathbf{c}[\mathrm{k}]$ & $k$-th underlay user's specific signature \\
$\overline{\mathbf{d}}[\bar{k}]$ & $k$-th overlay user's multiplexed data vector \\
$\mathbf{d}[k]$ & $k$-th underlay user's multiplexed data vector \\
$\mathbf{d}_{h}$ & Hybrid data vector \\
$G$ & Overlay spreading factor \\
$\mathbf{H}_{s s}$ & Secondary transmitter to secondary receiver channel matrix \\
$\mathbf{H}_{p s}$ & Primary transmitter to secondary receiver channel mtrix \\
$K$ & Total number of secondary users \\
$\bar{K}$ & Number of overlay secondary users \\
$K$ & Number of underlay secondary users \\
$\bar{M}$ & Total number of subcarriers \\
$M_{p u}$ & Number of occupied subcarriers by all primary users \\
$M_{s u}$ & Number of available subcarriers for overlay cognitive users \\
$\mathbf{n}[i]$ & Complex Gaussian noise component on the $i$-th subcarrier \\
$P$ & Number of consecutive symbols sent simultaneously by each \\
& overlay user \\
$\mathbf{r}$ & Received signal vector \\
$\overline{\mathbf{S}}$ & Overlay scrambling matrix \\
$\underline{\mathbf{S}}$ & Underlay scrambling matrix \\
$\mathbf{s}_{p u}$ & Primary user data vector containing availability vector a \\
\hline
\end{tabular}

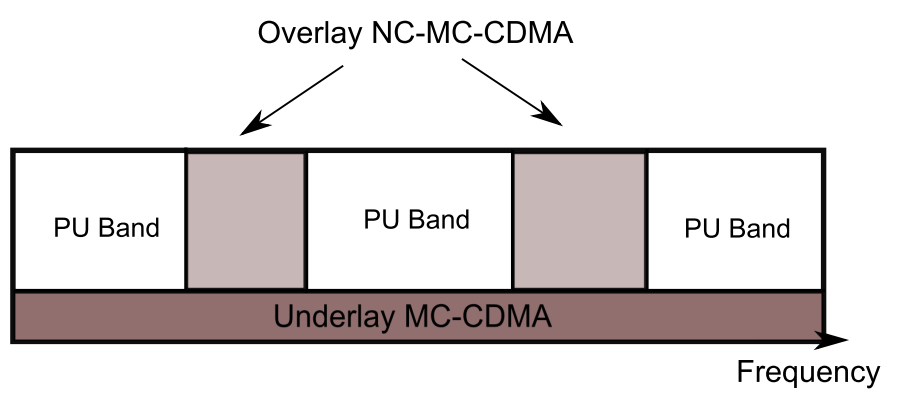

Fig. 1: Spectral occupancy of the hybrid MC-CDMA system

bands and the interference threshold for the occupied bands are known to the CRN. It is also assumed that the CR base station performs centralized control and allocates users into overlay and underlay transmissions based on their data rate requirements. By ensuring the underlay users transmit under the interference threshold, the interference to the PU is thus within the acceptable range and creates negligible performance degradation. As shown in Fig. 1, underlay is utilizing the whole spectrum while overlay is transmitting through the spectrum holes detected by the spectrum sensing unit. The number of subcarriers occupied by the PU system is represented by $M_{p u}$ and the number of subcarriers to be used by the overlay CR is shown by $M_{s u}$. The subcarrier availability for the CR system is shown by an $M$-element availability vector $\mathbf{a}$ in which $a_{i} \in\{0,1\}$ with 1 indicating the $i$-th subcarrier to be available, and 0 not available for the overlay. In this model, $\bar{K}$ overlay users are using the $M_{s u}$ available subcarriers and $\underline{K}$ underlay users will utilize the whole spectrum while maintaining the interference threshold of the PU and at the same time keeping the 


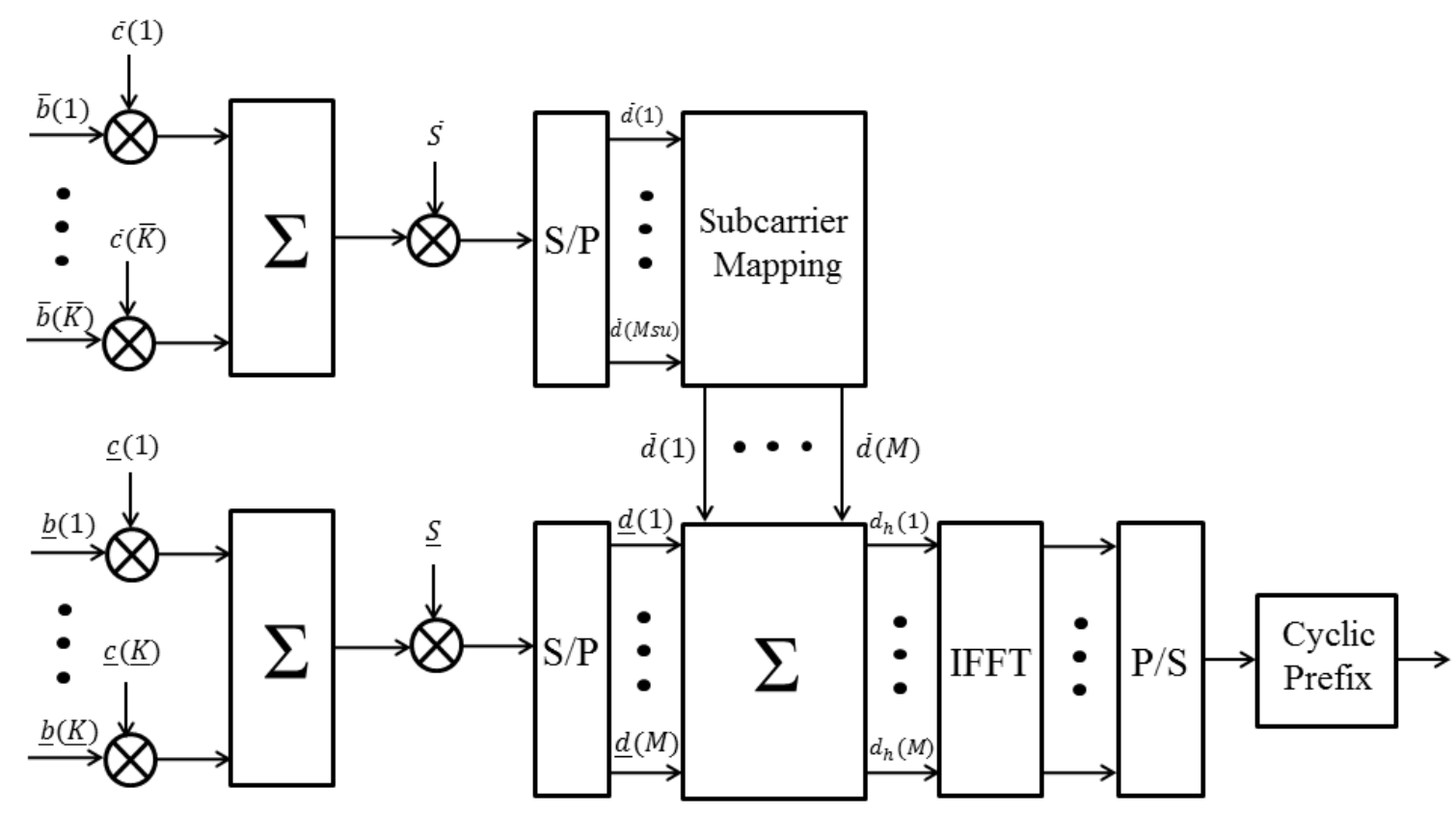

Fig. 2: Proposed Transmitter Structure

orthogonality with overlay users. The total number of cognitive users is denoted as $K^{1}$. $P$ consecutive symbols are spread with the spreading factor $G$ and are sent simultaneously by each overlay user, i.e. $M_{s u}=G P$. Since underlay is utilizing the whole spectrum, the underlay code length will be $M$. The spread data of the $\bar{k}$-th overlay user is obtained by multiplying the user's symbols by its specific signature sequence as

$$
\begin{aligned}
& \overline{\mathbf{y}}[\bar{k}]=\overline{\mathbf{b}}[\bar{k}] \otimes \overline{\mathbf{c}}[\bar{k}] \\
& \quad=\left[b_{1} c_{1}, \ldots, b_{1} c_{G}, \ldots, b_{P} c_{1}, \ldots, b_{P} c_{G},\right]^{T} \in \mathbb{C}^{M_{s u} \times 1}
\end{aligned}
$$

where $\overline{\mathbf{b}}[\bar{k}]$ of size $P \times 1$ is the $\bar{k}$-th user's symbol vector and $\overline{\mathbf{c}}[\bar{k}]$ of size $G \times 1$ is the $\bar{k}$-th user's specific spreading code. We denote $\overline{\mathbf{x}} \in \mathbb{C}^{M \times 1}$ as the equivalent overlay signal of $\overline{\mathbf{y}}$ after respective subcarrier mapping (according to the availability vector a) and summation over all $\bar{K}$ overlay users. The overlay spread data is then multiplied by the $M \times M$ overlay diagonal scrambling matrix $\overline{\mathbf{S}}$, which contains the $M_{s u}$-element scrambling sequence after the same subcarrier mapping as in $\overline{\mathbf{x}}$. The overlay multiplexed symbol vector of $\bar{K}$ users is then

$$
\overline{\mathbf{d}}=\overline{\mathbf{S}} \overline{\mathbf{x}} .
$$

The transmitter block diagram is shown in Fig. 2. The $k$-th underlay user's data symbol is represented by $\underline{b}[\underline{k}]$ and its $M \times 1$ spreading code is $\underline{\mathbf{c}}[\underline{\mathrm{k}}]$. The combined underlay

\footnotetext{
${ }^{1}$ In order to make it easier to follow, in this model a special notation is employed to address variables related to overlay and underlay. Any variable related to overlay is shown by an overline and any underlay variable is shown by an underline.
}

spread signal of $\underline{K}$ users is

$$
\underline{\mathbf{x}}=\sum_{\underline{k}=1}^{\underline{K}} \underline{\mathrm{b}}[\underline{k}] \underline{\mathbf{c}}[\underline{k}] .
$$

It is further multiplied by the underlay diagonal scrambling matrix $\underline{\mathbf{S}}$ of $M$ elements. Therefore, the transmitted hybrid signal can be shown by

$$
\mathbf{d}_{h}=\overline{\mathbf{S}} \overline{\mathbf{x}}+\underline{\mathbf{S}} \underline{\mathbf{x}}=\overline{\mathbf{d}}+\underline{\mathbf{d}}
$$

which is an $M \times 1$ vector consisting of the summation of overlay and underlay signals. The channel state information is assumed to be known perfectly at the receiver side, but not at the transmitter. Let

$$
\mathbf{H}_{s s}=\operatorname{diag}\left[\mathbf{h}_{s s}[1], \mathbf{h}_{s s}[2], \ldots, \mathbf{h}_{s s}[M]\right]
$$

be the diagonal $M \times M$ frequency domain complex channel from the secondary transmitter to the secondary receiver, where $\mathbf{h}_{s s}[i]$ is the channel gain on the $i$-th subcarrier. Likewise,

$$
\begin{aligned}
\mathbf{H}_{p s}=\operatorname{diag}\left[\left(1-a_{1}\right) \mathbf{h}_{p s}[1],\right. & \left(1-a_{2}\right) \mathbf{h}_{p s}[2], \ldots, \\
& \left.\left(1-a_{M}\right) \mathbf{h}_{p s}[M]\right]
\end{aligned}
$$

is the $M \times M$ frequency domain complex channel from the primary transmitter to the secondary receiver. Here, the unoccupied subcarriers will be set to zero by the term $\left(1-a_{i}\right)$. The channel is assumed to be frequency selective Rayleigh fading, with flat fading over each subcarrier. Then, the received signal on the $i$-th subcarrier at the secondary receiver is given by

$$
\mathbf{r}[i]=\mathbf{h}_{s s}[i] \mathbf{d}_{h}[i]+\mathbf{h}_{p s}[i] \mathbf{s}_{p u}[i]+\mathbf{n}[i]
$$


where

$$
\begin{array}{r}
\mathbf{s}_{p u}=\left[\left(1-a_{1}\right) \mathbf{d}_{p u}[1],\left(1-a_{2}\right) \mathbf{d}_{p u}[2], \ldots,\right. \\
\left.\left(1-a_{M}\right) \mathbf{d}_{p u}[M]\right]^{T}
\end{array}
$$

is the $M$ by 1 PU data matrix. The first part in (7) is the secondary user's hybrid received signal on the $i$-th subcarrier. The second term is the interference from PU on the $i$-th subcarrier and the last part, $\mathbf{n}[i]$, is the noise component on the $i$-th subcarrier of the received signal and is assumed to be complex Gaussian. The received signal in (7) can be expressed in vector form as

$$
\mathbf{r}=\mathbf{H}_{s s} \mathbf{d}_{h}+\mathbf{H}_{p s} \mathbf{s}_{p u}+\mathbf{n} .
$$

\section{Proposed Full-LoAd Hybrid Model}

\section{A. Transmitter}

In this model the number of cognitive users is equal to the overlay spreading factor to achieve full load transmission (i.e. $K=G$ ). The number of overlay users is $\bar{K}=G-1$ while one underlay user transmits through the entire bandwidth with respect to the interference threshold of the PU. Overlay utilizes the spectrum holes while maintaining orthogonality with underlay by using the Orthogonal Variable Spreading Factor (OVSF) codes. Spreading factor of $G$ is used for overlay users to spread the data symbols while the underlay user uses the code with length $M$ for spreading. In Fig. 2, $\overline{\mathbf{S}}$ and $\underline{\mathbf{S}}$ are diagonal matrices of 1 in this case, as the number of users will not be more than the signal's dimension and therefore, there is no need for scrambling.

\section{B. Receiver}

The receiver for the Full-load model detects independently the overlay and underlay users' signals. In other words, overlay performance does not affect underlay. Therefore, this model is preferable for the cases when the primary user's activity is high. Let us define $\mathbf{C}_{h} \in \mathbb{C}^{M \times K}$ as the hybrid spreading code matrix where the first to the $\bar{K}$-th rows belong to the overlay users and the last row is related to the underlay user which is of length $M$. Overlay spreading sequence is assumed to be periodic with period $G$, i.e. $c_{i+G, k}=c_{i, k}$. Next, the respective PU subcarriers of overlay users in $\mathbf{C}_{h}$ are set to zero.

Upon receiving the signal and removing cyclic prefix, Fast Fourier Transform (FFT) is applied. Cyclic prefix length is chosen such that it is longer than the maximum delay spread of the channel to avoid Inter-Carrier Interference. Passing through an equalizer, the signal on the $i$-th subcarrier can be shown as

$$
\mathbf{y}[i]=\mathbf{w}[i] \mathbf{r}[i]=\mathbf{w}[i] \mathbf{h}_{s s}[i] \mathbf{d}_{h}[i]+\mathbf{w}[i] \mathbf{h}_{p s}[i] \mathbf{s}_{p u}[i]+\mathbf{w}[i] \mathbf{n}[i]
$$

where $\mathbf{w}$ is the equalizer weight vector of size 1 by $M$, and $\mathbf{w}[i]$ is the equalizer's $i$-th coefficient. In the following, the two modified chip-level and symbol-level Minimum Mean
Squared Error (MMSE) equalization [19] is proposed and the performance is compared with Zero Forcing (ZF) results in Section V.

1) ZF Receiver: By multiplying the reciprocal of the channel, and despreading using the orthogonal spreading codes, ZF equalizer forces the Multi-Access Interference (MAI) component to zero. Therefore, the underlay decision variable consists of the desired signal, PU interference and noise which can be shown as

$$
\begin{aligned}
z_{u n}^{z f}= & \underline{\mathbf{b}}+\sum_{i=1}^{M} \mathbf{w}_{Z F}[i] \mathbf{h}_{p s}[i] \mathbf{C}_{h}[i, K] \mathbf{s}_{p u}[i] \\
& +\sum_{i=1}^{M} \mathbf{w}_{Z F}[i] \mathbf{C}_{h}[i, K] \mathbf{n}[i]
\end{aligned}
$$

where $\mathbf{C}_{h}[i, K]$ is the underlay user's $i$-th chip and $\mathbf{w}_{Z F}[i]$ is the reciprocal of the $S U$ transmitter to $S U$ receiver channel on the $i$-th subcarrier i.e. $\mathbf{w}_{Z F}[i]=1 / \mathbf{h}_{s s}[i]$. The underlay instantaneous Signal to Interference plus Noise Ratio (SINR) for ZF is

$$
\gamma_{u n}^{z f}=\frac{M p_{s_{u}}}{N_{0} \sum_{i=1}^{M}|\mathbf{w}[i]|^{2}+p_{p u} \sum_{i=1}^{M_{p u}}\left|\mathbf{w}[i] \mathbf{h}_{p s}[i]\right|^{2}}
$$

where $p_{p u}$ is the average PU symbol energy on each subcarrier and $p_{s_{u}}$ is the underlay symbol energy.

2) Chip Level MMSE: Chip-level equalization minimizes the mean square error between the transmitted signal and the estimated signal of each subcarrier. The despreading process is performed on each user's signal afterwards. It is a low-complexity single user detection method. The MMSE criterion for the $i$-th subcarrier is

$$
\arg \min _{\mathbf{w}_{C L}[i]} \mathbb{E}\left[\left|\mathbf{w}_{C L}[i] \mathbf{r}[i]-\underline{\mathbf{d}}[i]\right|^{2}\right] .
$$

Substituting (4) and (7) into the MMSE criterion (13), it can be easily shown that the MMSE-FDE on the $i$-th underlay subcarrier is given by (14) where $p_{c_{o}}$ is the overlay signal energy per chip. As mentioned earlier, it is assumed that there is no overlap for overlay and PU's band, while the underlay is orthogonal to the overlay. As a result, the conventional MMSE-FDE can be used for overlay signal detection [20]. Hence, the overlay signal detection is not analyzed here.

After recombining the signal over all subcarriers across the whole bandwidth, the underlay signals' decision variable with Chip-Level MMSE (CL-MMSE) is

$$
\begin{aligned}
& z_{u n}^{C L-M M S E}=\underline{\mathbf{b}} \sum_{i=1}^{M} \mathbf{w}_{C L}[i] \mathbf{h}_{s s}[i]+\sum_{k=1}^{\bar{K}} \beta^{(k, K)} \overline{\mathbf{b}}\left[\left\lceil\frac{i}{G}\right\rceil, k\right] \\
& +\sum_{i=1}^{M}\left\{\mathbf{w}_{C L}[i] \mathbf{h}_{p s}[i] \mathbf{C}_{h}[i, K] \mathbf{s}_{p u}[i]+\mathbf{w}_{C L}[i] \mathbf{C}_{h}[i, K] \mathbf{n}[i]\right\}
\end{aligned}
$$

where $\beta^{(k, K)}=\sum_{i=1}^{M} \mathbf{w}_{C L}[i] \mathbf{h}_{s s}[i] \mathbf{C}_{h}[i, k] \mathbf{C}_{h}[i, K]$ and $\lceil l\rceil$ denotes smallest integer not less than $l$. The first 


$$
\mathbf{w}_{C L}[i]=\frac{\mathbf{h}_{s s}^{*}[i]}{\mathbf{h}_{s s}[i] \mathbf{h}_{s s}^{*}[i]+\frac{N_{0}}{p_{c_{u}}}+\frac{p_{c_{o}}}{p_{c_{u}}} a_{i} \mathbf{h}_{s s}[i] \mathbf{h}_{s s}^{*}[i]+\frac{p_{p u}}{p_{c_{u}}}\left(1-a_{i}\right) \mathbf{h}_{p s}[i] \mathbf{h}_{p s}^{*}[i]}
$$

component in (15) contains the desired underlay signal. The second term is the MAI from overlay users due to the residual interference from MMSE equalization. The third term is the interference from the PU, and the last part is the noise component. Assuming Gaussian approximation, the underlay noise plus interference power can be written as ${ }^{2} \sigma_{\text {Tot }}^{2}=\sigma_{I_{p u}}^{2}+\sigma_{\bar{I}}^{2}+\sigma_{n}^{2}$. The variance of the AWGN component corresponds to

$$
\sigma_{\mathbf{I}_{n}}^{2}=\frac{N_{0}}{M} \sum_{i=1}^{M}\left|\mathbf{w}_{C L}[i]\right|^{2} .
$$

The variance of the PU interference will be

$$
\sigma_{\mathbf{I}_{p u}}^{2}=\frac{p_{p u}}{M} \sum_{i=1}^{M}\left|\mathbf{w}_{p u}[i] \mathbf{h}_{p s}[i]\right|^{2}
$$

where PU to SU channel coefficients are weighted by $\mathbf{w}_{p u}$

$$
\mathbf{w}_{p u}[i]=\frac{\left(1-\mathbf{a}_{i}\right) \mathbf{h}_{s s}^{*}[i]}{\frac{N_{0}}{p_{c_{u}}}+\mathbf{h}_{s s}[i] \mathbf{h}_{s s}^{*}[i]+\frac{p_{p u}}{p_{c_{u}}} \mathbf{h}_{p s}[i] \mathbf{h}_{p s}^{*}[i]} .
$$

Note that the summation's upper limit in (17) is $M$. However, the unoccupied subcarriers are set to zero by the term $\left(1-\mathbf{a}_{i}\right)$ in (18). The overlay interference variance is

$$
\begin{aligned}
\sigma_{\bar{I}}^{2} & =\operatorname{var}\left[\sum_{k=1}^{\bar{K}-1} \overline{\mathbf{b}}\left[\left\lfloor\frac{i}{G}\right\rfloor, k\right] \sum_{i=1}^{M} \mathbf{w}_{C L}[i] \mathbf{h}_{s s}[i] \mathbf{C}_{h}[i, k] \mathbf{C}_{h}[i, K]\right] \\
& =\frac{P(\bar{K}-1) p_{s_{o}}}{\sqrt{M \cdot G}} \sigma_{w_{C L} h_{s s}}^{2}
\end{aligned}
$$

where $p_{s_{o}}$ is the overlay symbol power and

$$
\sigma_{w_{C L} h_{s s}}^{2}=\mathbb{E}\left[\mathbf{w}_{C L}^{2} \mathbf{h}_{s s}^{2}\right]-\mathbb{E}^{2}\left[\mathbf{w}_{C L} \mathbf{h}_{s s}\right]
$$

is the variance of the SU to SU channel coefficients

$$
\mathbf{w}_{C L}[i]=\frac{\mathbf{a}_{i} \mathbf{h}_{s s}^{*}[i]}{\frac{N_{0}}{p_{c_{u}}}+\left(1+\frac{p_{c_{o}}}{p_{c_{u}}}\right) \mathbf{h}_{s s}[i] \mathbf{h}_{s s}^{*}[i]} .
$$

3) Symbol Level MMSE: Symbol-level equalization considers equalization and despreading jointly and hence minimizes the mean square error between the transmitted and estimated symbol at the expense of higher complexity. The MMSE criterion for underlay symbol is

$$
\min _{\mathbf{w}} \mathbb{E}\left[\left|z_{u n}-\underline{\mathrm{b}}\right|^{2}\right]=\min _{\mathbf{w}} \mathbb{E}\left[\left|\mathbf{w}_{S L} \mathbf{r}-\underline{\mathrm{b}}\right|^{2}\right]
$$

\footnotetext{
${ }^{2}$ Note that the variances are all conditional variances to channel coefficients $\left(\mathbf{H}_{s s}\right.$ and $\left.\mathbf{H}_{p s}\right)$ which is not shown here for notational simplicity.
}

Substituting the received vector, $\mathbf{r}$, from (4) and (9) into criterion (22), and differentiating with respect to $\mathbf{w}_{S L}^{*}$, the optimal vector can be shown as

$$
\begin{aligned}
\mathbf{w}_{S L}= & P_{s_{u}} \mathbf{c}_{h_{K}}^{H} \mathbf{H}_{s s}^{H} . \\
& \left(\mathbf{H}_{s s} \mathbf{C}_{h} \mathbf{R}_{d_{h}} \mathbf{C}_{h}^{H} \mathbf{H}_{s s}^{H}+\mathbf{H}_{p s} \mathbf{R}_{p p} \mathbf{H}_{p s}^{H}+\mathbf{R}_{n n}\right)^{-1}
\end{aligned}
$$

where $\mathbf{R}_{d_{h}}=\mathbb{E}\left[\mathbf{d}_{h} \mathbf{d}_{h}^{H}\right]$ is a $K \times K$ diagonal matrix of the users' symbol energy (i.e. the last element is the underlay user's symbol energy and the rest are overlay's), $\mathbf{R}_{p p}=\mathbb{E}\left[\mathbf{s}_{p u} \mathbf{s}_{p u}^{H}\right]$ and $\mathbf{R}_{n n}=\mathbb{E}\left[\mathbf{n} \mathbf{n}^{H}\right]=N_{0} I_{M} . \mathbf{c}_{h_{K}}$ is the $K$-th code of the hybrid code matrix $\mathbf{C}_{h}$ of size $M$ by 1 . The underlay signals' decision variable with Symbol-Level MMSE (SL-MMSE) is

$$
z_{u n}^{S L-M M S E}=\mathbf{w}_{S L} \mathbf{r}=\mathbf{w}_{S L} \mathbf{H}_{s S} \mathbf{s}+\mathbf{w}_{S L} \mathbf{H}_{p s} \mathbf{s}_{p u}+\mathbf{w}_{S L} \mathbf{n}
$$

which includes the desired signal and residual interference from overlay users, PU interference, and noise respectively. SL considers the non-diagonal elements in the equalization process while the chip-level ignores. This is why the MAI vanishes with symbol-level detection and equalization. Assuming the MAI component to be zero for symbol-level equalization, the underlay SINR can be written as

$$
\gamma_{u n}^{S L}=\frac{p_{c_{u}} \mathbf{w}_{S L} \mathbf{H}_{s s} \mathbf{H}_{s s}^{H} \mathbf{w}_{S L}^{H}}{\mathbf{w}_{S L} \mathbf{H}_{p s} \mathbf{s}_{p u} \mathbf{s}_{p u}^{H} \mathbf{H}_{p s}^{H} \mathbf{w}_{S L}^{H}+N_{0} \mathbf{w}_{S L} \mathbf{w}_{S L}^{H}} .
$$

Therefore with the proposed method, overlay will not have interference on underlay and hence the PU interference will be suppressed by a factor of $\frac{M}{M_{p u}}$.

\section{Proposed Overload Hybrid Model}

In this model the overlay users are in full load and the number of underlay users can be even larger than 1 and thus cheieve an overload transmission, depending upon the interference threshold of the PU system. Two layered spreading is performed, namely channelization and scrambling [21]. Overlay channelization code is Walsh-Hadamard (WH) of length $G$ while the underlay code is WH of length $M$. The use of WH codes ensures the orthogonality amongst the overlay users, and similarly for underlay users. However, since the overlay system is already fully loaded, the underlay user is overloading the system, and thus create interference. It should be mentioned that the overload system concept in cognitive radio has some elemental differences with conventional spread spectrum based overloaded systems. Firstly, the underlay interference threshold limit should be concerned at all times. Moreover, the overload user, transmitting via underlay, does not occupy the same number of subcarriers 
as overlay users. Therefore, the scrambling sequences should be updated according to the number of overlay available subcarriers from the spectrum sensing unit. The scrambling codes should also be updated when a new user is being added to the underlay hybrid system to choose the ones that create the least correlation with the overlay system.

\section{A. Code Allocation Algorithm}

The scrambling code is generated as follows. In order to achieve low cross-correlation between the overlay and underlay users, the orthogonal Gold codes are employed [22]. A pair of Gold codes of length $M-1$ is chosen. By appending a 0 at the tails of these two codes, two orthogonal Gold codes of length $M$ are generated, one for overlay and one for underlay. The part of the overlay scrambling code in which PUs exist is set to zero. The underlay scrambling code is then cyclic shifted and the one that provides the least cross-correlation with overlay users is selected for underlay scrambling. The algorithm can be written as follows where it is based on the average cross-correlation values than their maximum values [23]:

1) Generate a pair of orthogonal Gold codes of length $M$ for overlay and underlay scrambling.

2) Generate the periodic overlay channelization code $\overline{\mathbf{C}}$ for $\bar{K}$ users i.e. $\overline{\mathbf{C}}$ will be a $\bar{K} \times M_{s u}$ matrix, where $M_{s u}$ is obtained from the spectrum sensing unit.

3) Calculate the combined overlay code for $\bar{K}$ users as $\overline{\mathbf{T}}=\overline{\mathbf{C}} \overline{\mathbf{S}}$ and at the same time zero pad at the PU occupied subcarriers ( $\overline{\mathbf{T}}$ will be a matrix of $\bar{K} \times M$ ).

4) For the specific underlay user calculate the combined underlay code as $\underline{\mathbf{T}}[\underline{\mathrm{k}}]=\underline{\mathbf{c}}[\underline{\mathrm{k}}] \underline{\mathbf{S}}$ ( $\underline{\mathbf{T}}$ will be a $1 \times M$ vector).

5) Calculate the $\underline{k}$-th underlay user's correlation with the $\bar{k}$-th overlay user

$$
\Psi_{\underline{k}, \bar{k}, 0}=\frac{1}{M_{s u}} \sum_{i=1}^{M} \underline{\mathbf{T}}[i] \overline{\mathbf{T}}[\bar{k}, i]
$$

where the last index 0 denotes the number of cyclic shift of the underlay scrambling code and in this case is 0 .

6) Perform chip-wise cyclic-shift of the underlay scrambling code and repeat steps 3-5. The correlation for each shift is $\Psi_{k, \bar{k}, m}$ where $m \in\{1, \ldots, M\}$.

7) Repeat steps 3-6 for all underlay users and overlay users.

8) Choose the amount of shift that has the minimum correlation between the overlay and underlay users' scrambling codes

$$
\hat{m}=\arg \min _{m} \frac{1}{\underline{k}} \sum_{i=1}^{\underline{K}}\left(\frac{1}{\bar{k}} \sum_{j=1}^{\bar{K}} \Psi_{i, j, m}\right) .
$$

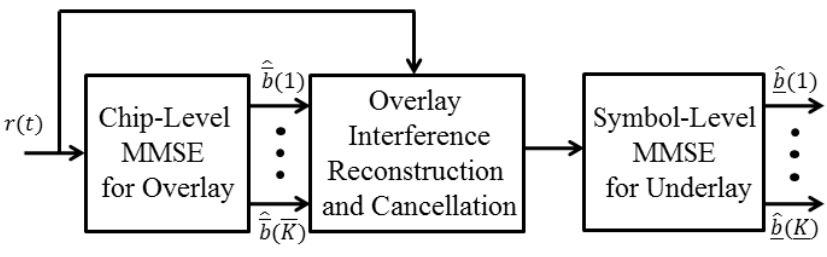

Fig. 3: Overload Receiver Block Diagram

\section{B. Receiver}

The block diagram of the proposed receiver is shown in Fig. 3. The received signal is descrambled by using the overlay scrambling sequence. The overlay signal is first detected, due to its relative high power to the underlay signals, from the received hybrid signal using chip-level MMSE. There are two main reasons to use chip-level MMSE for overlay detection. Firstly, chip-level detector can maintain the simplicity of the MC-CDMA receiver for overlay users as it does not require the knowledge of other users' sequences. Secondly, since the overlay transmission power is considerably higher than that of the underlay and there is no interference from PU, the chip-level MMSE exhibits a good performance. The chip-level MMSE criterion for the $i$-th overlay subcarrier is given by

$$
\min _{\overline{\mathbf{w}}[i]} \mathbb{E}\left[|\mathbf{z}[i]-\overline{\mathbf{d}}[i]|^{2}\right]=\min _{\overline{\mathbf{w}}[i]} \mathbb{E}\left[|\overline{\mathbf{w}}[i] \mathbf{r}[i]-\overline{\mathbf{d}}[i]|^{2}\right]
$$

where $\mathbf{z}_{i}$ is the decision variable on the $i$-th subcarrier. Substituting (7), where the second term regarding PU is set to zero by $\mathbf{h}_{p s}[i]$ for overlay subcarrier, into (28) and differentiating with respect to $\overline{\mathbf{w}}^{*}$ [24], it can be easily shown that the chip-level equalization coefficient for overlay is

$$
\overline{\mathbf{w}}[i]=\frac{\mathbf{h}_{s s}^{*}[i]}{\left(1+\frac{p_{c_{u}}}{p_{c_{o}}}\right) \mathbf{h}_{s s}[i] \mathbf{h}_{s s}^{*}[i]+\frac{N_{0}}{p_{c_{o}}}} .
$$

After overlay descrambling and despreading, the overlay data is detected to be $[\hat{\bar{b}}[1], \hat{\bar{b}}[2], \ldots, \hat{\bar{b}}[\bar{K}]]$. Therefore, after overlay interference reconstruction and cancellation, the modified received signal on the $i$-th subcarrier for underlay detection is

$$
\hat{\mathbf{r}}[i]=\mathbf{r}[i]-\sum_{i=1}^{M} \hat{\overline{\mathbf{x}}}[i] \mathbf{h}_{s s}[i]
$$

where $\hat{\overline{\mathbf{x}}}[i]$ is the sum of $\bar{K}$ overlay users' detected multiplexed data on the $i$-th subcarrier. Therefore, the reconstructed received signal component after overlay signal detection and cancellation corresponds to

$$
\hat{\mathbf{r}}_{c}=\mathbf{H}_{s s} \underline{\mathbf{d}}+\mathbf{H}_{p s} \mathbf{s}_{p u}+\mathbf{n}+\hat{\overline{\mathbf{I}}}
$$

where $\hat{\overline{\mathbf{I}}}$ is the residual interference from overlay due to imperfect cancellation. It is assumed to be zero in the 
subsequent derivation due to the relative high overlay to underlay power. In order for the underlay signals to be detected under the high interference from the PUs, symbol-level equalization is considered for underlay as it has better performance than chip-level equalizer. The MMSE criterion for underlay is

$$
\min _{\underline{\mathbf{W}}} \mathbb{E}\left[(\underline{\mathbf{W r}}-\underline{\mathbf{b}})(\underline{\mathbf{W}} \mathbf{r}-\underline{\mathbf{b}})^{H}\right]
$$

The optimal vector for this objective function, as shown in the Appendix, is

$$
\begin{aligned}
\underline{\mathbf{W}}= & \mathbf{R}_{\underline{b b}} \underline{\mathbf{C}}^{H} \underline{\mathbf{S}}^{H} \mathbf{H}_{s s}^{H} . \\
& \left(\mathbf{H}_{s s} \underline{\mathbf{S}} \underline{\mathbf{C}} \mathbf{R}_{\underline{b}} \underline{\mathbf{C}}^{H} \underline{\mathbf{S}}^{H} \mathbf{H}_{s s}^{H}+\mathbf{H}_{p s} \mathbf{R}_{p p} \mathbf{H}_{p s}^{H}+\mathbf{R}_{n n}\right)^{-1}
\end{aligned}
$$

where $\mathbf{R}_{b b}=\mathbb{E}\left[\underline{b b}{ }^{H}\right]$ is a $\underline{K} \times \underline{K}$ diagonal matrix of the underlay users' symbol energy ${ }^{3}$. The proposed algorithm in Section IV-A chooses the least correlated codes between overlay and underlay. On the other hand, utilizing SL-MMSE detection for underlay minimizes the MAI after encountering fading environment. In addition, knowing that overlay to underlay power is considerably higher, the MAI can be assumed to be negligible. Therefore, the underlay SINR can be written as

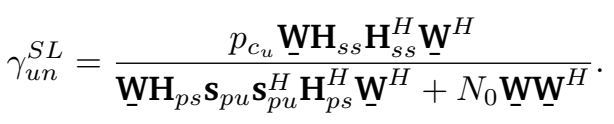

Since several symbols are sent through overlay in each block, any potential overlay error will not directly propagate and make underlay erroneous. On the other hand, in case that the overlay performance is poor, the proposed Full-load method will be preferable.

\section{Simulation RESUlts}

This section presents the simulation results and compares the proposed systems' performance for different scenarios with existing systems. The total available bandwidth is assumed to be $10 \mathrm{MHz}$ and chip duration is 100ns. The channel between PU to SU and SU to PU are modelled by the ITU-Pedestrian B [25]. A total of 512 subcarriers in 8 blocks of 64 are available for the PU system. The primary system uses OFDMA and each user occupies one block of 64 consecutive subcarriers. If a block is not occupied by the PU, it will be exploited by the overlay users with a spreading factor of 64, i.e., each subcarrier carries one chip. The underlay is spreading the data over the whole 512 subcarriers respecting the interference threshold of the PU. Two scenarios are considered: Medium and High PU to underlay interference level. Finally, the Multi-User underlay scenario is presented in Section V-C.

\footnotetext{
${ }^{3}$ Note that underlay signal power $\left(P_{u n}=\operatorname{tr}\left(\mathbf{R}_{b b}\right)\right)$ has been set to be lower than the PU's interference threshold $\left(I_{T}\right)$ i.e. $\left(P_{u n} \leq B I_{T}\right)$.
}

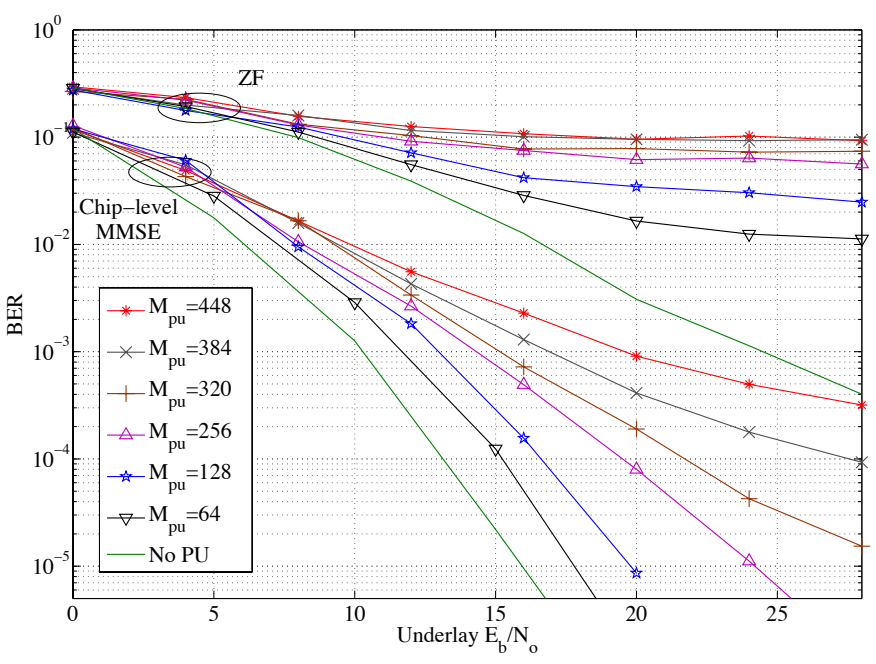

Fig. 4: Underlay performance of the proposed full-load hybrid system with ZF and chip-level MMSE equalizers for different PU occupancy levels

\section{A. Medium PU interference level}

For the Full-load case, there are a total of 64 cognitive users in the system. Overlay users are utilizing the unoccupied spectrum in chunks of 64 subcarriers. The last user is transmitting in underlay over the total PU bandwidth. In this scenario, the secondary user's underlay received power is assumed to be $-20 \mathrm{~dB}$ relative to the received signal power from the PU whilst it is maintained below the PU interference threshold. Overlay to underlay relative power is also $20 \mathrm{~dB}$. Fig. 4 compares the underlay performance of the full-load system for ZF and chip-level MMSE at different PU occupancy levels. The underlay performance is shown for $M_{p u}=64,128,256,320,384$ and 448 subcarriers being occupied by the PU system which accounts for $12.5 \%, 25 \%, 50 \%, 62.5 \%, 75 \%$ and $87.5 \%$ of the total bandwidth respectively. The baseline error performance for ZF and MMSE with no PU interference, denoted by No PU in the figure, are also plotted. It can be observed that the proposed chip-level MMSE considerably enhances the underlay performance with low complexity. The underlay performance also improves with less PU occupancy due to reduced level of interference.

Fig. 5 compares the chip-level performance with the proposed symbol level MMSE for different PU occupancy levels. The solid lines show the symbol-level and dashed lines show the chip-level performance. It is observed that the symbol-level equalization results in a significant BER performance improvement for all PU occupancy levels. For instance, symbol-level MMSE equalization at $M_{p u}=320$ achieves a $5 \mathrm{~dB}$ improvement over chip level at $10^{-4}$ BER. It should also be noted that at $M_{P U}=64$, the symbol level equalizer performs close to the No PU case, effectively suppressing the PU interferences. On a separate note, the effect of interference between overlay and underlay users are also evaluated, and simulation 
results show that overlay users suffer no degradation from the underlay users as they are orthogonal. The

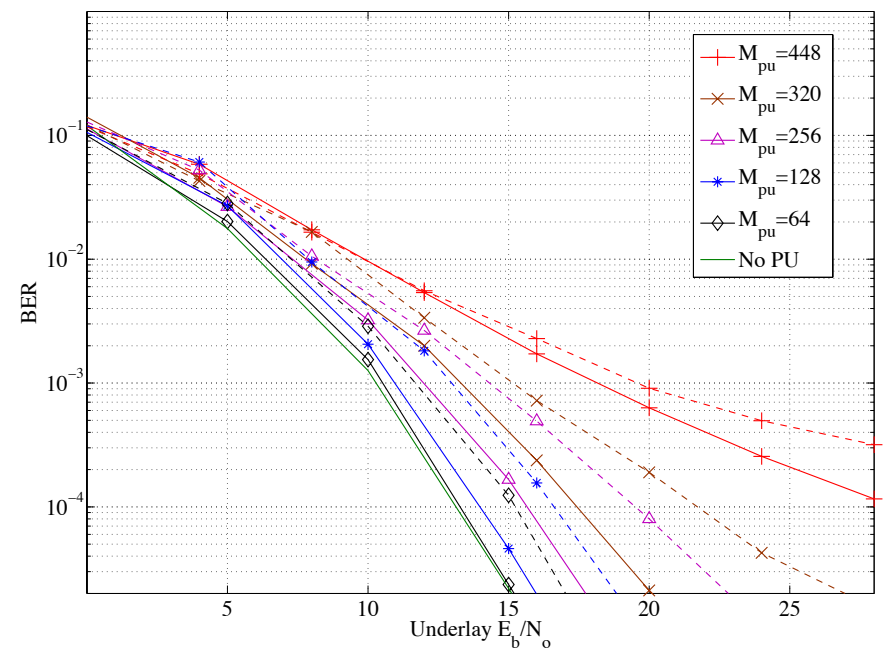

Fig. 5: Underlay performance of the proposed full-load hybrid system with chip and symbol level MMSE equalizers for different PU occupancy levels and with relative underlay to $\mathrm{PU}$ received interference level of -20 $\mathrm{dB}$; Dashed and solid lines represent chip and symbol level performances respectively.

underlay performance is also evaluated against different number of overlay users. As orthogonal codes are used, the performance is unchanged for $\mathrm{ZF}$ and symbol level equalizer, with a slight degradation in chip level due to the residual interference in MMSE. For brevity, these results are omitted. Fig. 6 compares the underlay performance results for the full-load and overload systems when the relative overlay to underlay and PU to underlay powers are kept at $20 \mathrm{~dB}$, as in the previous scenario. For the overload system, the overlay is fully loaded, i.e. 64 users in blocks of 64 subcarriers, and one underlay user is transmitting through underlay. The scrambling codes are selected using the algorithm explained in Section IV-A. It is observed that for high PU occupancy levels the overload system's performance diverges more from the full-load case while for low PU occupancy the performance of the two proposed systems converge. This is because when PU occupancy level is low, the overloaded underlay signal will have most of the interference coming from the overlay users, which can be suppressed due to the scrambling code. However when PU occupies more subcarriers, the underlay user suffers from higher interference and due to overloaded operations, the interference rejection capability is weakened comparing to the full load case. However it must be noted that the BER plot in Figure 6 slightly favours the full load operation, as it has a lower total rate comparing to the overloaded case (due to one less user).

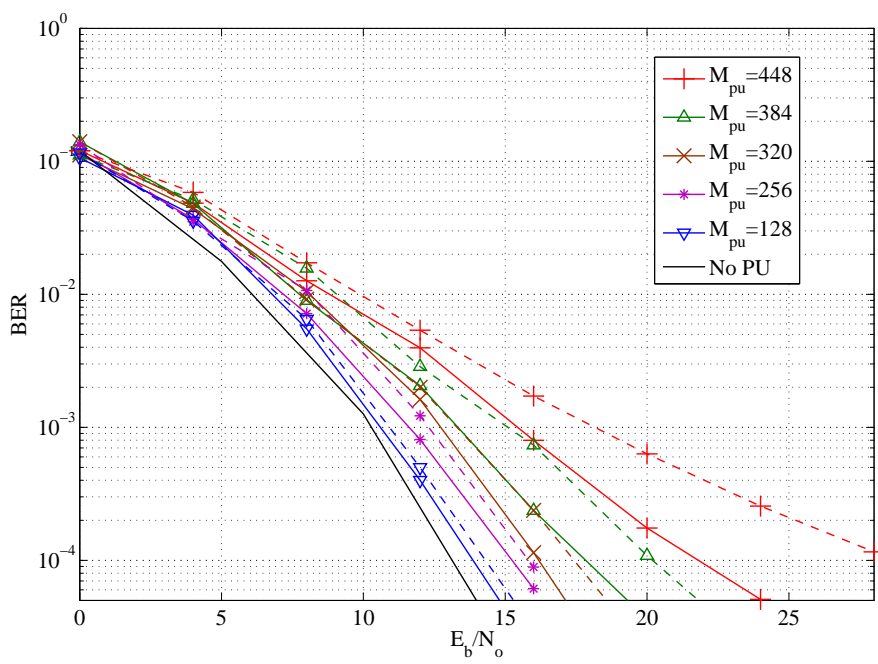

Fig. 6: Proposed full-load and Overload underlay performance comparison with relative underlay to PU received interference level of $-20 d B$; Dashed and solid lines represent full-load and overload performances respectively.

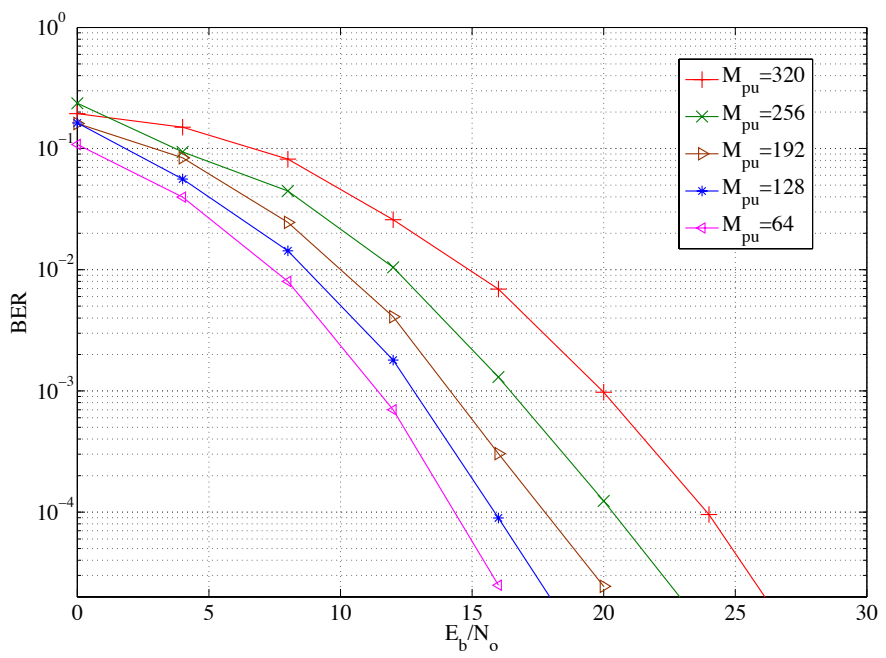

Fig. 7: Underlay performance of the proposed hybrid overload system with different PU occupancy levels and high PU interference level.

\section{B. High PU Interference level}

In this scenario, the interference from the $\mathrm{PU}$ is increased to $44 \mathrm{~dB}$ relative to the underlay received power while interference threshold is kept at the same level as in the previous part. The overlay to underlay power is $47 \mathrm{~dB}$. The underlay BER performance of the proposed overload system is presented for different number of PU occupancy levels, $M_{p u}=64,128,192,256$ and 320, in Fig. 7. The results show that in spite of very high interference level from PU, the underlay maintains good performance and as the number of available overlay subcarriers increases, the underlay performance enhances. 


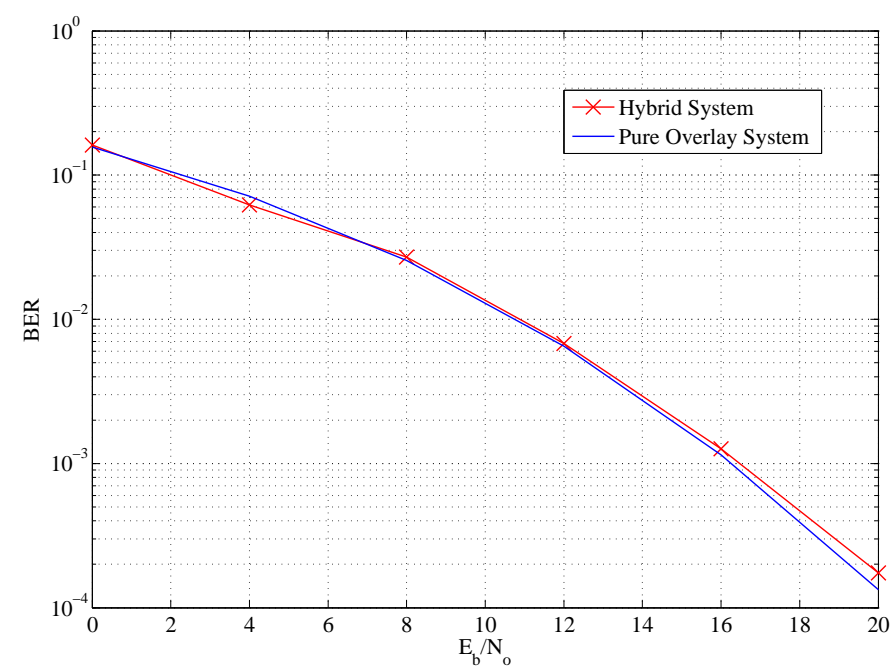

Fig. 8: Overlay performance with and without underlay transmission.

Next, the effect of underlay transmission to the overlay users in the proposed overloaded operation is evaluated. Unlike previous simulations where the overlay was assumed to be transmitting with significantly higher power relative to underlay, in this part the overlay and underlay are transmitting at the same power. This is the worst case scenario for the overlay users to evaluate the worst possible performance. The overlay BER performance is compared to that of an overlay-only system and is depicted in Fig. 8. In the hybrid case, overlay occupancy level is $50 \%$ (256 subcarriers). It is observed that the overlay performance degradation is very small. Therefore, with the proposed hybrid system the underlay can enhance the spectral efficiency without degrading the overlay performance.

The underlay sensitivity of the proposed system due to PU interference power is shown in Fig. 9. In this scenario, the number of overlay subcarriers is fixed to 256 . The PU received power at the secondary receiver is varying while the interference threshold and hence the underlay power is kept the same. It is observed that increasing the PU interference power from $37 \mathrm{~dB}$ to $44 \mathrm{~dB}$, the underlay performance is degraded by $2 \mathrm{~dB}$ or less. This shows the robustness of the overloaded system performs well in high PU interference scenario.

Fig. 10 compares the NC-MC-CDMA underlay approach [15] with the proposed overload performance. For the NC-MC-CDMA case, underlay is sending in the PU occupied parts of the spectrum only, i.e. 256 subcarriers. The Dashed lines show the NC-MC-CDMA and solid lines show the proposed systems results. It is observed that with increasing the PU interference, the performance of the NC-MC-CDMA underlay degrades dramatically while the proposed system still maintains good results, in addition to more transmission rate. For instance, for PU interference level of $44 \mathrm{~dB}$, the proposed system still shows

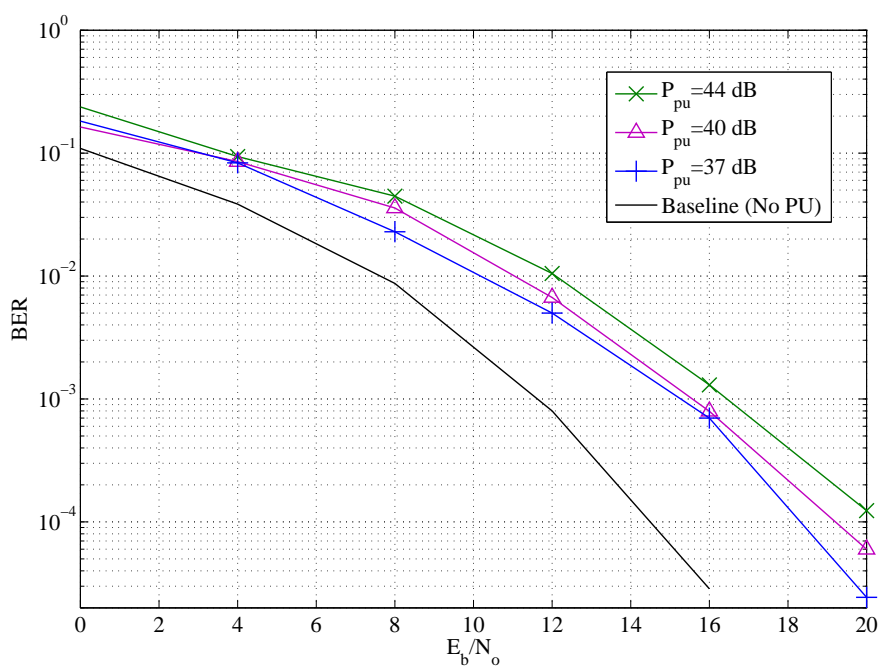

Fig. 9: Underlay sensitivity of the proposed overload system to PU interference power level, $M_{p u}=256$

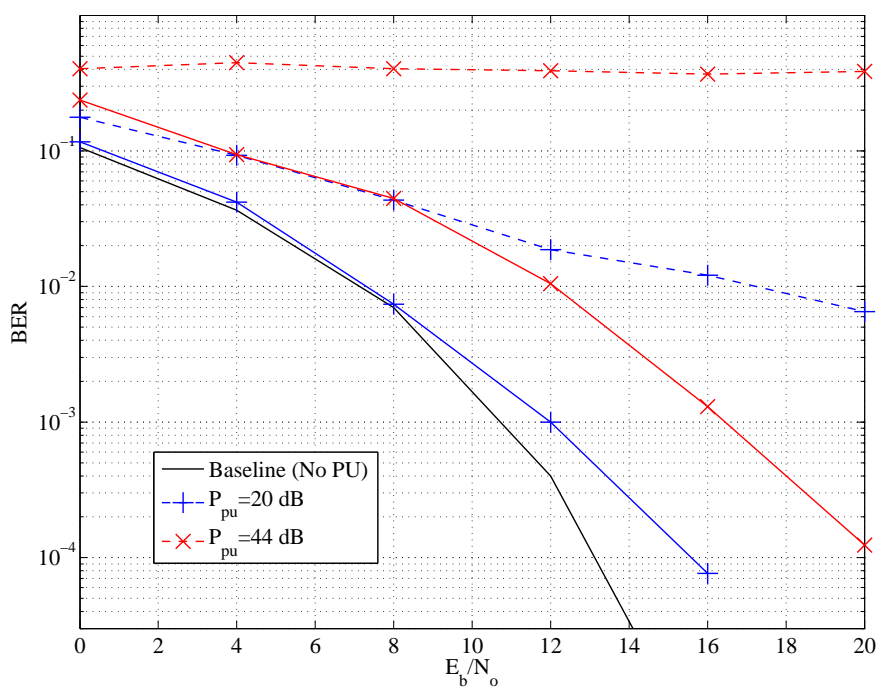

Fig. 10: Underlay NC MC-CDMA sensitivity to PU interference power level for 256 subcarriers. Dashed lines show the NC-MC-CDMA performance and solid lines the proposed system's results with $M_{p u}=256$.

better result than the previous NC-MC-CDMA of $20 \mathrm{~dB}$.

\section{Underlay Multi-user results}

In this part, the overload system with multiple underlay users is considered. Clearly, the number of underlay users depend on the PU interference threshold and also the (Quality of Service) QoS requirement of the SU. But to evaluate the proposed code assignment algorithm, the interference threshold is assumed to increase as the number of underlay users increases. This way, the underlay degradation due to underlay multi access interference can be evaluated.

Fig. 11 presents the underlay performance with increasing number of underlay users for two cases 


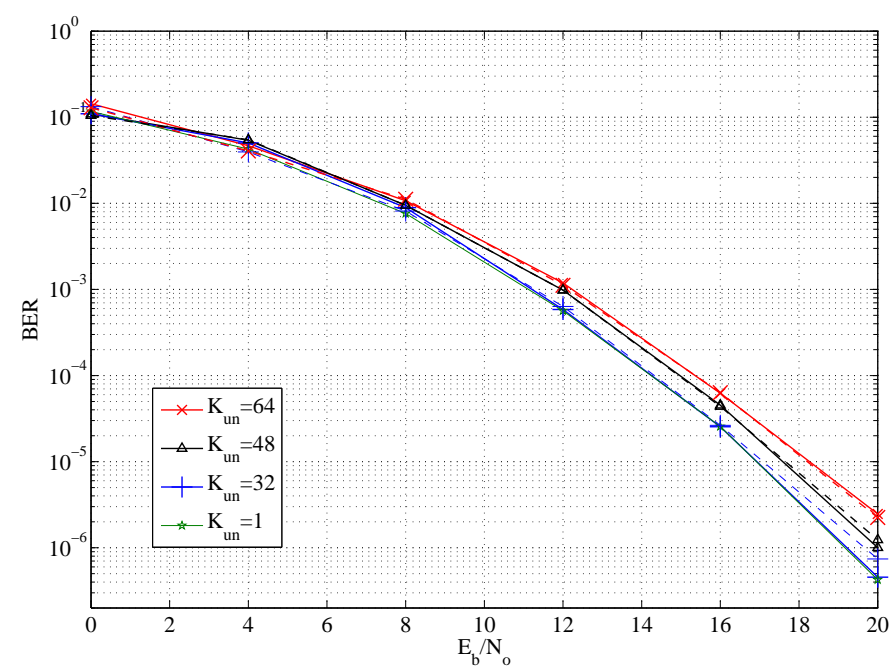

Fig. 11: Underlay performance for increasing number of underlay users for two cases when overlay is fully loaded and when there is no overlay user in the system; $M_{p u}=$ 64 .

of full-overlay and without overlay users, using solid and dashed lines respectively. The number of occupied subcarriers, $M_{p u}$, is considered to be 64 , i.e. PU is utilizing one block of subcarriers only and the rest of the spectrum is being utilized by overlay cognitive system. The number of underlay users, $\underline{K}$, is shown by $K_{u n}$ in the figure. Underlay interference to overlay is assumed to be negligible since the overlay transmission power is considerably higher than the underlay one. On the other hand, the underlay codes have been chosen meticulously and according to the number of overlay and underlay overlapping subcarriers to make the least possible correlation with the overlay system.

Several conclusions can be made from the figure. Firstly, it is observed that using the proposed code selection algorithm, the underlay performance degradation due to MAI with increasing number of underlay users is negligible for $50 \%$ overload (i.e., $\underline{K}=G / 2$ or below). Note that the total number of CR users (both overlay and underlay) in this case can be up to $\frac{3}{2} G$. Therefore, the interference threshold determines how many underlay users can be added to the underlay hybrid system according to the users' requirements. Secondly, comparing the solid line and dashed line for each case, it is observed that the underlay performance degradation due to overlay is negligibly small for any number of underlay users. This shows the effectiveness of the scrambling code selection algorithm explained in Section IV-A in suppressing the interference between the overlay and underlay users.

\section{CONCLUSIONS}

In this paper two hybrid integrated MC-CDMA systems are proposed to enhance the spectral efficiency and interference mitigation in cognitive radio networks.
The first proposed system is a full-load MC-CDMA system that maintains orthogonality between overlay and underlay using OVSF codes. The modified MMSE chip-level and symbol-level equalizers are also proposed. The symbol-level scheme was shown to have better BER performance than the chip-level in all PU occupancy levels, at the expense of higher complexity. The benefit with the full-load system is that the underlay works independently from overlay, i.e., it does not need to detect overlay symbols in order to detect underlay data. The second is an overload system that uses the full signal dimension for the overlay users, while the overload user will utilize the underlay transmission using a two layered spreading. The proposed underlay BER performance for the overload and full-load scenarios are compared. It is shown that the overload outperform the existing in addition to its higher data rate. The system is then extended to a multi-user underlay system. Simulation results show that using the proposed code selection algorithm, the underlay performance degradation due to MAI with increasing number of underlay users is negligible for up to $50 \%$ overload.

\section{APPENDIX}

Substituting $\hat{\mathbf{r}}_{c}$ from (31) into the objective function of (32), knowing that $\underline{\mathbf{d}}=\underline{\mathbf{S}} \underline{\mathbf{C}} \underline{\mathbf{b}}$,

$$
\begin{aligned}
& \mathbf{J}=E\left[\left(\left(\underline{\mathbf{W}} \mathbf{H}_{s s} \underline{\mathbf{S}} \underline{\mathbf{C}} \underline{\mathbf{b}}+\underline{\mathbf{W H}}_{p s} \mathbf{s}_{p u}+\mathbf{W} \mathbf{n}\right)-\underline{\mathbf{b}}\right)\right. \\
& \left.\left(\left(\mathbf{W H}_{s s} \underline{\mathbf{S}} \underline{\mathbf{C}}+\underline{\mathbf{W}}_{p s} \mathbf{S}_{p u}+\underline{\mathbf{W}}\right)-\underline{\mathbf{b}}\right)^{H}\right] \\
& =\underline{\mathbf{W H}}_{s s} \underline{\mathbf{S}} \underline{\mathbf{R}}_{\underline{b b}} \underline{\mathbf{C}}^{H} \underline{\mathbf{S}}^{H} \mathbf{H}_{s s}^{H} \underline{\mathbf{W}}^{H}-\mathbf{R}_{b b} \underline{\mathbf{W}} \mathbf{H}_{s s} \underline{\mathbf{S}} \underline{\mathbf{C}} \\
& +\underline{\mathbf{W H}}_{p s} \mathbf{R}_{p p} \mathbf{H}_{p s}^{H} \underline{\mathbf{W}}^{H}+\mathbf{W R}_{n n} \mathbf{W}^{H} \\
& -\mathbf{R}_{\underline{b b}} \underline{\mathbf{C}}^{H} \underline{\mathbf{S}}^{H} \mathbf{H}_{s s}^{H} \underline{\mathbf{W}}^{H}+\mathbf{R}_{\underline{b b}} .
\end{aligned}
$$

Differentiating the above expression with respect to $\underline{\mathbf{w}}^{H}$ and set it to 0 i.e $\frac{d \mathbf{J}}{d \underline{\mathbf{w}}^{H}}=0$

$$
\begin{aligned}
\Rightarrow & \underline{\mathbf{w}} \mathbf{H}_{s s} \mathbf{S}_{u n} \mathbf{C}_{u n} \mathbf{R}_{\underline{b b}} \mathbf{C}_{u n}^{H} \mathbf{S}_{u n}^{H} \mathbf{H}_{s s}^{H}+\mathbf{w H}_{p s} \mathbf{R}_{p p} \mathbf{H}_{p s}^{H} \\
& +\underline{\mathbf{w}}_{n n}-\mathbf{R}_{\underline{b b}} \mathbf{C}_{u n}^{H} \mathbf{S}_{u n}^{H} \mathbf{H}_{s s}^{H}=0 .
\end{aligned}
$$

Rearranging for $\underline{\mathbf{w}}$, the proof is completed.

\section{REFERENCES}

[1] J. Tadrous, A. Sultan, and M. Nafie, "Admission and power control for spectrum sharing cognitive radio networks," IEEE Trans. Wireless Commun., vol. 10, no. 6, pp. 1945-1955, June 2011.

[2] L. B. Le and E. Hossain, "Resource allocation for spectrum underlay in cognitive radio networks," IEEE Trans. Wireless Commun., vol. 7, no. 12, pp. 5306-5315, December 2008.

[3] K. Son, B. C. Jung, S. Chong, and D. K. Sung, "Opportunistic underlay transmission in multi-carrier cognitive radio systems," in IEEE Wireless Commun. Net. Conf. (WCNC), April 2009, pp. 1 $-6$.

[4] Q. Qu, L. Milstein, and D. Vaman, "Cognitive radio based multiuser resource allocation in mobile ad hoc networks using multicarrier cdma modulation," IEEE J. Select. Areas in Commun., vol. 26, no. 1, pp. 70-82, Jan 2008.

[5] T. Weiss and F. Jondral, "Spectrum pooling: an innovative strategy for the enhancement of spectrum efficiency," IEEE Mag. Commun., vol. 42, no. 3, pp. S8 - 14, Mar 2004. 
[6] B. Farhang-Boroujeny and R. Kempter, "Multicarrier communication techniques for spectrum sensing and communication in cognitive radios," IEEE Mag. Commun., vol. 46, no. 4, pp. 80-85, April 2008.

[7] G. Bansal, M. Hossain, and V. Bhargava, "Optimal and suboptimal power allocation schemes for ofdm-based cognitive radio systems," IEEE Trans. Wireless Commun., vol. 7, no. 11, pp. $4710-4718$, november 2008

[8] — - "Adaptive power loading for OFDM-based cognitive radio systems with statistical interference constraint," IEEE Trans. Wireless Commun., vol. 10, no. 9, pp. 2786 -2791, september 2011.

[9] A. Attar, M. Nakhai, and A. Aghvami, "Cognitive radio transmission based on direct sequence MC-CDMA," IEEE Trans. Wireless Commun., vol. 7, no. 4, pp. 1157 -1162, April 2008.

[10] FCC, "Federal communications commission spectrum policy task force: Report of the spectrum efficiency working group," Nov. 2002.

[11] S. Haykin, "Cognitive radio: brain-empowered wireless communications," IEEE J. Sel. Areas in Commun., vol. 23, no. 2, pp. $201-220$, Feb. 2005.

[12] L. B. Le and E. Hossain, "Resource allocation for spectrum underlay in cognitive radio networks," IEEE Trans. Wireless Commun., vol. 7, no. 12, pp. 5306-5315, 2008.

[13] I. Akyildiz, W. Lee, M. Vuran, and S. Mohanty, "Next generation/dynamic spectrum access/cognitive radio wireless networks: a survey," Computer Networks, vol. 50, no. 13, pp. 21272159, 2006.

[14] G. Bansal, M. Hossain, V. Bhargava, and T. Le-Ngoc, "Subcarrier and power allocation for OFDMA-based cognitive radio systems with joint overlay and underlay spectrum access mechanism," IEEE Trans. Veh. Technol., vol. 62, no. 3, pp. 1111-1122, 2013.

[15] V. Chakravarthy, X. Li, R. Zhou, Z. Wu, and M. Temple, "Novel overlay/underlay cognitive radio waveforms using SD-SMSE framework to enhance spectrum efficiency-part II: analysis in fading channels," IEEE Trans. Commun., vol. 58, no. 6, pp. 1868 -1876 , June 2010.

[16] V. Chakravarthy, X. Li, Z. Wu, M. Temple, F. Garber, R. Kannan, and A. Vasilakos, "Novel overlay/underlay cognitive radio waveforms using SD-SMSE framework to enhance spectrum efficiency- part I: theoretical framework and analysis in AWGN channel," IEEE Trans. Commun., vol. 57, no. 12, pp. 3794 -3804, December 2009.

[17] G. Ganesan and Y. Li, "Cooperative spectrum sensing in cognitive radio networks," in Proc. IEEE Dynamic Spectrum Access Networks, pp. 137-143, 2005.

[18] W. Prawatmuang, D. So, and E. Alsusa, "Sequential cooperative spectrum sensing technique in time varying channel," Wireless Communications, IEEE Transactions on, vol. 13, no. 6, pp. 33943405, June 2014.

[19] A. Phasouliotis and D. So, "Layered space-time receiver for downlink multiple-input multiple-output multi-carrier code division multiple access systems," IET Commun., vol. 5, no. 13, pp. 1907-1917, 2011.
[20] A. Goldsmith, Wireless communications. Cambridge Univ. Pr, 2005.

[21] E. Dinan and B. Jabbari, "Spreading codes for direct sequence CDMA and wideband CDMA cellular networks," IEEE Commun. Mag., vol. 36, no. 9, pp. 48-54, 1998.

[22] L. Hanzo, M. Munster, B. J. Chio, and T. Keller, OFDM and MC-CDMA for broadband multi-user communication, WLANs and broadcasting. John Wiley and Sons, 2003.

[23] I. Oppermann and B. Vucetic, "Complex spreading sequences with a wide range of correlation properties," IEEE Trans. Commun., vol. 45, no. 3, pp. 365-375, Mar 1997.

[24] A. Hjorungnes and D. Gesbert, "Complex-valued matrix differentiation: Techniques and key results," IEEE Trans. Signal Process., vol. 55, no. 6, pp. 2740-2746, 2007.

[25] "Selection procedures for the choice of radio transmission technologies of the UMTS (UMTS 30.03 version 3.2.0),," Tech. Rep., 101112 V3.2.0, 1998-04.

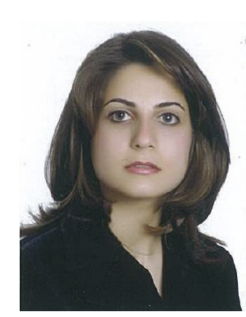

Fahimeh Jasbi received her BSc and MSc in Electrical and Electronic Engineering in 2005 and 2008 respectively from Azad University, Tehran, Iran. She received her $\mathrm{PhD}$ degree from University of Manchester, UK in 2014. Her research interests include congitive radio, $5 \mathrm{G}$ networks, interference cancellation, multiple access techniques, channel equqlization and estimation, and channel coding techniques.

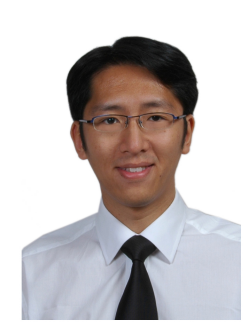

Daniel K. C. So (S'96-M'03-SM14) received the BEng degree in Electrical and Electronics Engineering (First Class Honor) from the University of Auckland, New Zealand in 1996, and the PhD degree in Electrical and Electronics Engineering from the Hong Kong University of Science and Technology (HKUST), Hong Kong in 2003.

From 1997 to 1998, he joined the Orion Systems International Limited, New Zealand, as a software engineer, and was later promoted to senior software engineer. Since 2003, he has been a lecturer and now a senior lecturer in the School of Electrical and Electronics Engineering, The University of Manchester, UK. He is also serving as the Director of Postgraduate Taught since 2009.

His research interests includes green communications, 5G networks, heterogeneous networks, cognitive radio, massive MIMO, D2D communications, cooperative MIMO schemes, multihop communication, channel equalization and estimation techniques. He regularly serves on the technical program committee of major international conferences. 